\title{
Acute Traumatic Brain Injury-Induced Neuroinflammatory Response and Neurovascular Disorders in the Brain
}

\author{
Duraisamy Kempuraj ${ }^{1,2,3}$ - Mohammad Ejaz Ahmed 1,2,3 . Govindhasamy Pushpavathi Selvakumar ${ }^{1,2,3}$. \\ Ramasamy Thangavel ${ }^{1,2,3} \cdot$ Sudhanshu P. Raikwar ${ }^{1,2,3} \cdot$ Smita A. Zaheer $^{1,2}$. Shankar S. Iyer ${ }^{1,2,3}$. \\ Raghav Govindarajan ${ }^{1}$ • Premkumar Nattanmai Chandrasekaran ${ }^{1} \cdot$ Casey Burton ${ }^{4}$. Donald James ${ }^{4}$. \\ Asgar Zaheer ${ }^{1,2,3}$ (1]
}

Received: 16 August 2020 / Revised: 12 September 2020 / Accepted: 14 September 2020 / Published online: 21 September 2020

(C) Springer Science+Business Media, LLC, part of Springer Nature 2020

\begin{abstract}
Acute traumatic brain injury (TBI) leads to neuroinflammation, neurodegeneration, cognitive decline, psychological disorders, increased blood-brain barrier (BBB) permeability, and microvascular damage in the brain. Inflammatory mediators secreted from activated glial cells, neurons, and mast cells are implicated in the pathogenesis of TBI through secondary brain damage. Abnormalities or damage to the neurovascular unit is the indication of secondary injuries in the brain after TBI. However, the precise mechanisms of molecular and ultrastructural neurovascular alterations involved in the pathogenesis of acute TBI are not yet clearly understood. Moreover, currently, there are no precision-targeted effective treatment options to prevent the sequelae of TBI. In this study, mice were subjected to closed head weight-drop-induced acute TBI and evaluated neuroinflammatory and neurovascular alterations in the brain by immunofluorescence staining or quantitation by enzyme-linked immunosorbent assay (ELISA) procedure. Mast cell stabilizer drug cromolyn was administered to inhibit the neuroinflammatory response of TBI. Results indicate decreased level of pericyte marker platelet-derived growth factor receptor-beta (PDGFR- $\beta$ ) and BBB-associated tight junction proteins junctional adhesion molecule-A (JAM-A) and zonula occludens-1 (ZO-1) in the brains 7 days after weight-drop-induced acute TBI as compared with the brains from sham control mice indicating acute TBI-associated BBB/ tight junction protein disruption. Further, the administration of cromolyn drug significantly inhibited acute TBI-associated decrease of PDGFR- $\beta$, JAM-A, and ZO- 1 in the brain. These findings suggest that acute TBI causes BBB/tight junction damage and that cromolyn administration could protect this acute TBI-induced brain damage as well as its long-time consequences.
\end{abstract}

Keywords Blood-brain barrier · Neuroinflammation · Tight junction proteins $\cdot$ Traumatic brain injury

Duraisamy Kempuraj

duraisamyk@health.missouri.edu

$\triangle$ Asgar Zaheer

Zaheera@health.missouri.edu

1 Department of Neurology, School of Medicine, University of Missouri, 1 Hospital Drive, Columbia, MO, USA

2 The Center for Translational Neuroscience, School of Medicine, University of Missouri, 1 Hospital Drive, Columbia, MO, USA

3 Harry S. Truman Memorial Veterans Hospital, U.S. Department of Veterans Affairs, Columbia, MO, USA

4 Phelps Health, Rolla, MO, USA

\section{Introduction}

Traumatic brain injury (TBI) is defined as "an alteration in brain function or other evidence of brain pathology caused by an external force (open/penetrating or closed/non-penetrating) (Brain Injury Association of America, Vienna, VA, USA)." TBI is a neuroinflammatory and neurodegenerative disorder. Most TBIs, such as concussion in the military, are mild TBI (mTBI). Even single mTBI/concussion can cause long-term consequences in the brain, including blood-brain barrier (BBB) damage, activation of glial cells, accumulation of tau, and neurofibrillary tangles (NFTs), dementia, and psychiatric disorders (Dinet et al. 2019; McKee and Robinson 2014). Many incidents, including falls, assaults, vehicle accidents, sports-related activities, head trauma, gunshots, occupation-associated injuries, child abuse, domestic violence 
activities, rapid acceleration and deceleration, military operations, and blasts, can cause TBI. TBI may be mild, moderate, or severe that differently impacts the sequelae of TBI. Nearly 1.7 million Americans are involved in TBI every year in the USA (Ma et al. 2019). We and others have shown that TBI causes direct primary initial brain damage that affects neuronal cells, astrocytes, and blood vessels and then induce indirect secondary brain damage through neuroinflammatory response (Ahmed et al. 2020a, b; Akamatsu and Hanafy 2020; Charkviani et al. 2019; Kempuraj et al. 2019a, 2020; Raikwar et al. 2020; Selvakumar et al. 2020).

Changes in the neurovascular unit are the indication of secondary injuries in the brain after TBI. The secondary brain injury involves neuroimmune and inflammatory responses that may start within days, or weeks or months, or many years after the initial primary brain damage following TBI (Crupi et al. 2020; Ladak et al. 2019). Secondary brain injury is associated with reduced cerebral blood supply, increased blood-brain barrier (BBB) permeability, edema formation, oxidative stress, axonal and neuronal damage, infiltration of peripheral blood cells, activation of resident immunocytes, the release of chemotactic and inflammatory mediators, and neuronal loss. The acute or chronic effects of neurotrauma considerably vary from person to person and in animal models of TBI (Khellaf et al. 2019; Zysk et al. 2019). Early mast cell activation and mast cells are critical effectors in neurotrauma/ stroke (Arac et al. 2019; Jin et al. 2009; Kempuraj et al. 2019a, b, 2020a; Mattila et al. 2011; McKittrick et al. 2015).

We have also recently reported that mast cell activation plays a role in the neuroinflammatory response and BBB disruption in acute TBI in mice (Kempuraj et al. 2020). Cromolyn (cromolyn), a mast cell stabilizer, inhibits cerebral mast cell degranulation, increases postoperative BBB stability, and improves cognitive function after neurotrauma/ hypoxic-ischemic brain damage (Jin et al. 2007; Zhang et al. 2016). Another study reported that cromolyn reduced histamine release from mast cells after epilepticus-induced brain damage in rats (Valle-Dorado et al. 2015). Cromolyn administration reduced $\mathrm{BBB}$ breakdown and brain edema in cerebral artery occlusion-induced ischemia or ischemic stroke in rodents (Mattila et al. 2011; McKittrick et al. 2015). As cromolyn shows beneficial neuroprotective effects in neuroinflammation and neurotrauma, we have tested this drug in acute TBI mice. BBB consists of microvascular endothelial cells, pericytes, and astrocytes. Pericytes ensheath the capillary wall and are essential regulators of the stability and permeability of BBB (Bhowmick et al. 2019). BBB breach is an early sign of cognitive disorder, and minimizing BBB dysfunction could reduce TBI severity (Nation et al. 2019; Sivandzade et al. 2020). BBB dysfunction has been reported as early as $3 \mathrm{~min}$ after concussion (Dinet et al. 2019). Loss of pericyte is one of the hallmarks of BBB dysfunction that may lead to neuroinflammation and neurodegeneration (Choi et al.
2016; Sengillo et al. 2013). Damage and degeneration of pericyte, endothelial cells, astrocytes, tight junction proteins, and basement membrane cause memory and vascular dysfunctions, including BBB breakdown (He et al. 2020; Jullienne et al. 2016). Platelet-derived growth factor-beta (PDGF- $\beta$ ) released from endothelial cells binds to PDGF receptor- $\beta$ (PDGFR- $\beta$ ) on pericytes and mediate many functions, including the attachment of pericytes with microvascular endothelial cells and endothelial proliferation. PDGFR- $\beta$ is abundantly expressed in pericytes and is a reliable marker for pericytes (Sengillo et al. 2013). PDGFR- $\beta$ can attract pericytes to endothelial cells during the formation of BBB. However, the precise mechanism of BBB and vascular dysfunction is not yet understood in acute TBI. Thus, in this preliminary study, we have analyzed PDGFR- $\beta$, junctional adhesion molecule-A (JAM-A), and Zonula occludens-1 (ZO-1) expression in closed head weight-drop-induced acute TBI in mice. We report reduced levels/derangement of PDGFR- $\beta$, JAM-A, and ZO-1 in acute TBI brains in mice and that administration of cromolyn drug could protect TBIassociated brain damage.

\section{Materials and Methods}

\section{Reagents}

Cryoprotection solution was purchased from FD Neuro Technologies (Columbia, MD). Polyclonal glial fibrillary acidic protein (GFAP) antibody, Alexa Fluor 568 donkey anti-rabbit $(\mathrm{H}+\mathrm{L})$ antibody, 4',6-diamidino-2-phenylindole, dihydrochloride (DAPI), tissue extraction reagent, Dulbecco's phosphate-buffered saline (DPBS), BCA Protein Assay Kit (Pierce), and 1-step ultra TMB-ELISA substrate solution were obtained from Thermo Fisher Scientific (Rockford, IL). Cromolyn sodium salt (cromolyn; CR) drug and goat anti-mouse IgG FITs antibody was purchased from Sigma-Aldrich (St. Louis, MO). Anti-ZO1 tight junction protein antibody (Rabbit polyclonal antibody), anti-PDGFR- $\beta$ (mouse monoclonal antibody), anti-JAM-A /JAM-1 (Rabbit polyclonal antibody), and anti-NeuN antibody (Rabbit polyclonal antibody) for the neuronal marker were purchased from Abcam (Cambridge, MA). JAM-A DuoSet enzyme-linked immunosorbent assay (ELISA) kit was purchased from the R\&D System (Minneapolis, MN).

\section{Closed Head Weight-Drop-Induced Acute TBI in Mice}

Closed head acute TBI was induced in 8-week-old male mice (CD-1 mice; the University of Missouri, Mutant Mouse Resource and Research Center and the University of Missouri Animal Modeling Core, Columbia, MO) by standard weight-drop procedure, as reported previously with slight 
modifications (Milman et al. 2005; Shishido et al. 2019; Zohar et al. 2003; Zvejniece et al. 2019). Briefly, the mouse was first anesthetized with isoflurane, and then the head was positioned on a spongy cushioned surface. Immediately, the mouse skull was exposed on the top with a small incision. Then a metal weight of $35 \mathrm{~g}$ was allowed to fall from $80 \mathrm{~cm}$ above the head through a vertical tube and induced cortical contusion without craniotomy (Milman et al. 2005; Shishido et al. 2019; Zohar et al. 2003; Zvejniece et al. 2019). Only the skin incision was made and then sutured in sham control mice instead of the weight-drop procedure. TBI was induced on the left side of the skull, and this position is comparable in all the animals. Mice ( $n=6$ mice/group) were monitored daily for 7 days ( 7 D) and then euthanized by cervical dislocation. In a separate group of mice, mast cell inhibitor drug cromolyn $(50 \mathrm{mg} / \mathrm{kg}$ body weight in sterile Phosphate Buffered Saline; the Food and Drug Administration (FDA) approved mast cell stabilizer drug) was administered intraperitoneally $1 \mathrm{~h}$ before TBI, 2nd and 4th day after TBI for a total of three injections (Moretti et al. 2016). The brains were obtained from all the mice and processed for section cutting using a cryostat machine. Brain sections $(20 \mu \mathrm{m})$ were cut and stored frozen in cryoprotection solution Inc., at $-80^{\circ} \mathrm{C}$ until used for GFAP, NeuN, DAPI, PDGFR- $\beta$, JAM-A, and ZO-1 immunofluorescence staining (Kempuraj et al. 2018a, b). Maintenance of mice and the experiments was conducted "according to the recommendations in the guide for the care and use of laboratory animals of the National Institutes of Health (NIH) and the approval of the committee on the Ethics of Animal Experiments of the University of Missouri (Columbia, MO).”

\section{Detection of GFAP, NeuN, DAPI, PDGFR- $\beta$, JAM-A, and ZO-1 Levels in Acute TBI in Mice Brains by Immunofluorescence Staining}

The brain sections from 7 days of TBI brains and sham control mice brains were fixed with a $4 \%$ paraformaldehyde solution. Immunofluorescence staining was performed using antiGFAP polyclonal antibody (1:500), anti-NeuN rabbit polyclonal antibody (1:500), anti-PDGFR- $\beta$ (1:500), antibody, anti-JAM-A (1:500) and anti-ZO-1 (1:500), as we reported (Kempuraj et al. 2018a, b). Briefly, free-floating brain sections were incubated with primary antibodies at $4{ }^{\circ} \mathrm{C}$ overnight. These sections were then incubated with a mixture of Alexa Fluor 488 goat anti-rabbit $\operatorname{IgG}(1: 300)$ and Alexa Fluor 568 anti-mouse IgG/goat anti-mouse (1:500) or separately for $1 \mathrm{~h}$ in 12-well tissue culture plates on a plate shaker at room temperature. Cellular nuclei were identified with DAPI staining. Then these brain sections were mounted, dried, and observed under a confocal fluorescent microscope (Leica Microsystems $\mathrm{GmbH}$, Germany). Images were captured using an oil immersion objective $(40 \times$ or $63 \times$ ) (Ahmed et al. 2019; Kempuraj et al. 2018b; Raikwar et al. 2018; Selvakumar et al. 2018;
Thangavel et al. 2018). The staining intensity was quantified in the photomicrographs at three different areas using Image $\mathrm{J}$ software (National Institute of Health, Bethesda, MD). These results were presented as \% of sham control in the bar graphs.

\section{Quantification of JAM-A Level in the TBI Brain Tissue by ELISA}

JAM-A level was quantified in the brain tissue lysates. Tissue lysates were prepared from the brains of sham control mice and TBI mice using a tissue tearor unit (Biospec Products Inc. Racine, WI). Protein level was quantified in the tissue lysates using the BCA protein assay kit. JAM-A level was determined in the brain tissue lysates by ELISA as per the procedure mentioned in the kit. Lysates with $150 \mu \mathrm{g}$ of protein/sample were used for the assay. Finally, the optical density of the ELISA microplate was read at $450 \mathrm{~nm}$ using a microplate reader (Molecular Devices, Sunnyvale, CA), as we have previously reported (Kempuraj et al. 2020, 2018a, b).

\section{Behavioral Analysis in TBI Mice}

\section{Cognitive Function Analysis by Novel Object Recognition Test}

Novel object recognition (NOR) test is commonly done to determine visual memory and object recognition memory functions in the animal models of neurological diseases such as mouse models of TBI. Mouse explores a novel object more time as compared with a familiar object. The mouse with a cognitive disorder may not fully or sufficiently recognize familiar objects, and therefore may not spend more time near the novel objects. In the NOR test, the mouse was first exposed to two similar objects for $5 \mathrm{~min}$, a day before TBI to familiarize in an open field arena of a square plexiglass apparatus with high walls. The total amount of time spent close to each object was noted. Following this, the mouse was removed from the open field arena and put in its original cage. Next, the mouse was exposed to one familiar object as well as one novel object in the same open field arena after 7 days after the TBI. The time spent close to each object was noted and determined the cognitive memory status, as previously reported (Bader et al. 2019; Tadepalli et al. 2020).

\section{Data Analysis}

All the results were statistically analyzed by one-way analysis of variance (ANOVA) and Tukey-Kramer multiple comparison analysis using GraphPad InStat 3 software. An unpaired $t$ test was used when comparing only two groups. A significant difference between the TBI groups or with the Sham control group was determined. The results were presented as mean \pm SEM. A $p$ value of $<0.05$ was considered statistically significant in comparing the findings in this study. 


\section{Results}

\section{Characteristics of Acute TBI-Induced Neuroinflammation in Mice Brains}

To confirm the neuroinflammatory response in TBI (7 days), mice brains from sham control and TBI mice brains were analyzed for the activation of astrocytes. The presence of astrocyte marker GFAP was analyzed in the cortical region (Fig. 1, $n=5$ /mice group). Representative image of sham control brain and TBI brain showing damaged impact area (arrows) in the cortex (Fig. 1a), and photomicrographs show a significant increase of activated astrocytes, indicating the presence of astrogliosis and reactive astrocytes, as evidenced by the presence of increased levels of GFAP immunoreactivity in TBI (7 days) mice brains as compared with sham control mice brains (Fig. 1b, GFAP/astrocytes). Next, we analyzed the levels of NeuN to detect neuronal cells in these brains. Results show significantly decreased levels of NeuN in 7 days of TBI brains as compared with sham control mice brains (Fig. $1 b$, neurons). Cellular nuclei were stained with DAPI. Further, we assessed visual memory functions in TBI mice by the standard NOR test ( $n=6$ mice/group). Results showed that sham control mice spent more time at the novel object than at the familiar object (Fig. 1c, $* p<0.05$ ). However, TBI mice did not fully recognize the familiar object. These results show weight-drop-induced TBI activates astrocytes and reduces visual/spatial memory performance in mice.

\section{Acute TBI Decreases PDGFR- $\beta$ and JAM-A Levels in the TBI Brain}

PDGFR- $\beta$ and JAM-A levels were analyzed in the brains of 7 days following closed head weight-drop-induced TBI mice, TBI mice with cromolyn treatment, and sham control mice by immunofluorescence staining ( $n=5 /$ mice group). Representative photomicrographs show decreased levels of both PDGFR- $\beta$ (arrows) and JAM-A (arrows) in 7 days of acute TBI brains, as compared with sham control brains (Fig. 2). However, administration of cromolyn drug (three doses) to TBI mice showed inhibition of TBI-associated decrease of PDGFR- $\beta$ and JAM-A levels in the brains indicating
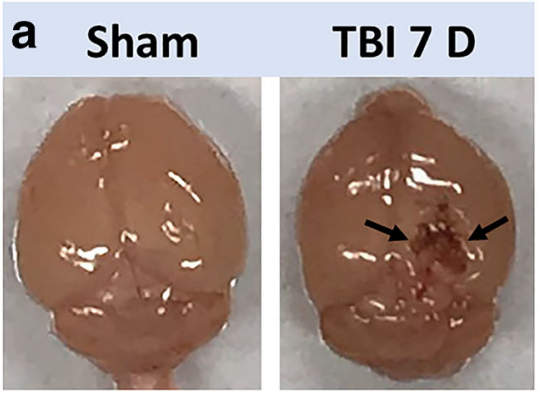

C

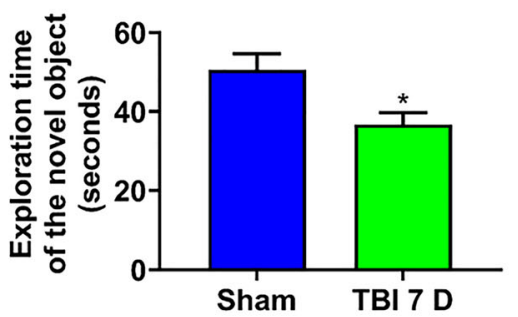

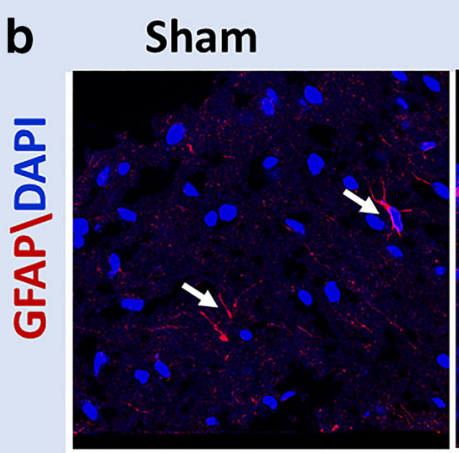

TBI 7 D

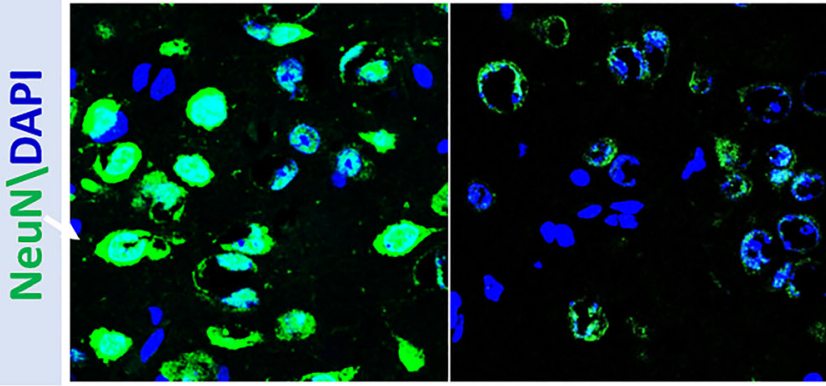

response after TBI. Cellular nuclei were stained with DAPI in these sections. Photomicrographs original magnifications $=630 \mathrm{x}$. c Next, we assessed memory function in TBI mice by the NOR test. In this test, the mouse was first exposed to two similar objects in an open field arena box for $5 \mathrm{~min}, 1$ day prior to TBI induction ( $n=6 \mathrm{mice} /$ group). The total amount of time spent near the objects was noted. Following this, the mouse was exposed to one familiar object and one novel object for 5 min. Then the time spent at each object was noted after 7 days of TBI. We found that sham control mice spent more time at the novel object than the familiar object as compared with TBI mice $(* p<0.05$, sham control vs. TBI 7 days). However, TBI mice did not recognize the familiar object. 7 D, 7 days 


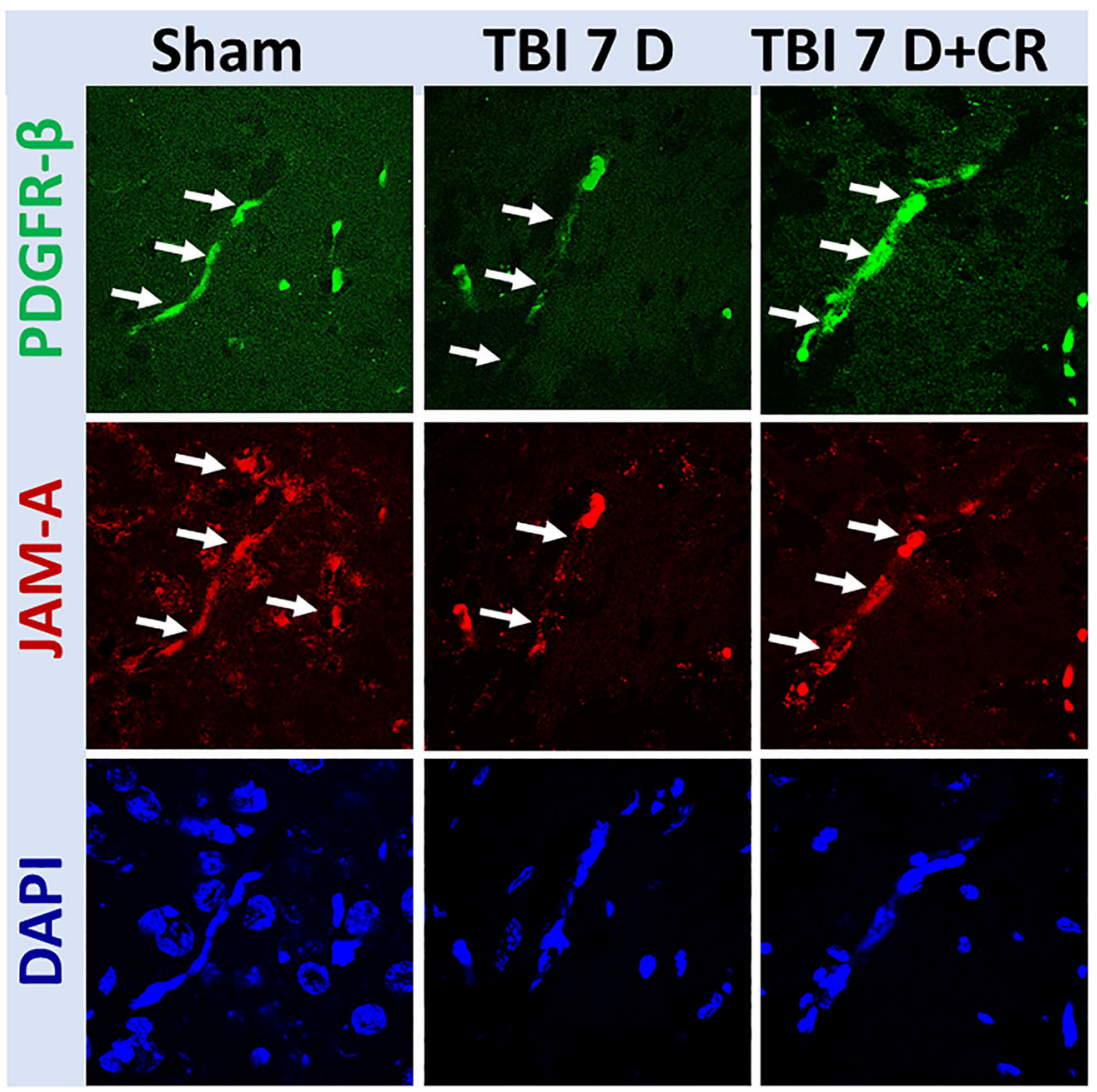

Fig. 2 Closed head weight-drop-induced acute TBI decreases PDGFR- $\beta$ and JAM-A levels in the brain. Pericyte marker PDGFR- $\beta$, and tight junction protein JAM-A levels were analyzed in the frozen sections $(20 \mu \mathrm{m})$ cut from 7 days following weight-drop-induced TBI, cromolyn administered mice, and sham control mice brains ( $n=5$ mice/group) by immunofluorescence staining using anti- PDGFR- $\beta$ and anti-JAM-A primary antibodies and Alexa Fluor 488 goat anti-rabbit IgG and Alexa Fluor 568 anti-mouse IgG/goat anti-mouse secondary antibodies.

a beneficial effect of the mast cell inhibitor (Fig. 2). The nuclei were stained with DAPI. These results suggest that closed head TBI induces degeneration/damage of pericytes as well as tight junction protein JAM-A in the brain.

\section{Acute TBI Affects ZO-1 Level in the TBI Brain}

We next analyzed the level of another tight junction protein, ZO-1, in the brains after 7 days of closed head acute weightdrop-induced TBI mice, Cromolyn administered mice and sham control mice by immunofluorescence staining (Fig. 3, $n=3$ mice/group). Representative images (Fig. 3a, arrows) and immunoreactivity intensity quantification bar graph (Fig. 3b, $* p<0.05 \mathrm{~B}$ ) show significant derangement/ decreased level of ZO-1 in 7 days of acute TBI brains, as compared with sham control mice brains. Cromolyn administration significantly prevented a decrease of the ZO-1 level in TBI mice brains, as compared with untreated TBI mice brains. The cellular nuclei were stained with DAPI.
Fluorescence images were captured using a confocal microscope. Representative images and immunoreactivity intensity bar graphs show decreased levels of both PDGFR- $\beta$ (arrows) and JAM-A (arrows) in 7 days of acute TBI brains as compared with sham control mice brains. However, cromolyn treatment in TBI (7 D) mice inhibited TBI-associated decrease of PDGFR- $\beta$ and JAM-A levels in the brain. The nuclei were stained with DAPI. Photomicrographs original magnifications $=\times 630.7$ D, 7 days; CR, cromolyn

\section{Cromolyn Drug Inhibits Acute TBI-Induced Decrease of Tight Junction Protein JAM-A Level in the Brain}

In addition to immunofluorescence staining, we have also quantified the JAM-A level in the brain tissue lysates of sham control mice, 7 days after TBI mice, and 7 days after TBI plus cromolyn treated mice ( $n=3$ mice/group) by ELISA. Our tight junction protein quantification results indicate a significantly decreased level of JAM-A in 7 days TBI mice brains as compared with sham control mice brains (Fig. 4 , $* p<0.05$ ). Further, the administration of mast cell inhibitor cromolyn drug significantly inhibited TBI-induced JAM-A decrease as compared with the levels in untreated TBI mice brains.

\section{Discussion}

In the present study, we observed reduced levels of pericyte marker PDGFR- $\beta$ and tight junction proteins JAM-A and ZO1 after 7 days of closed head weight-drop-induced TBI mice 

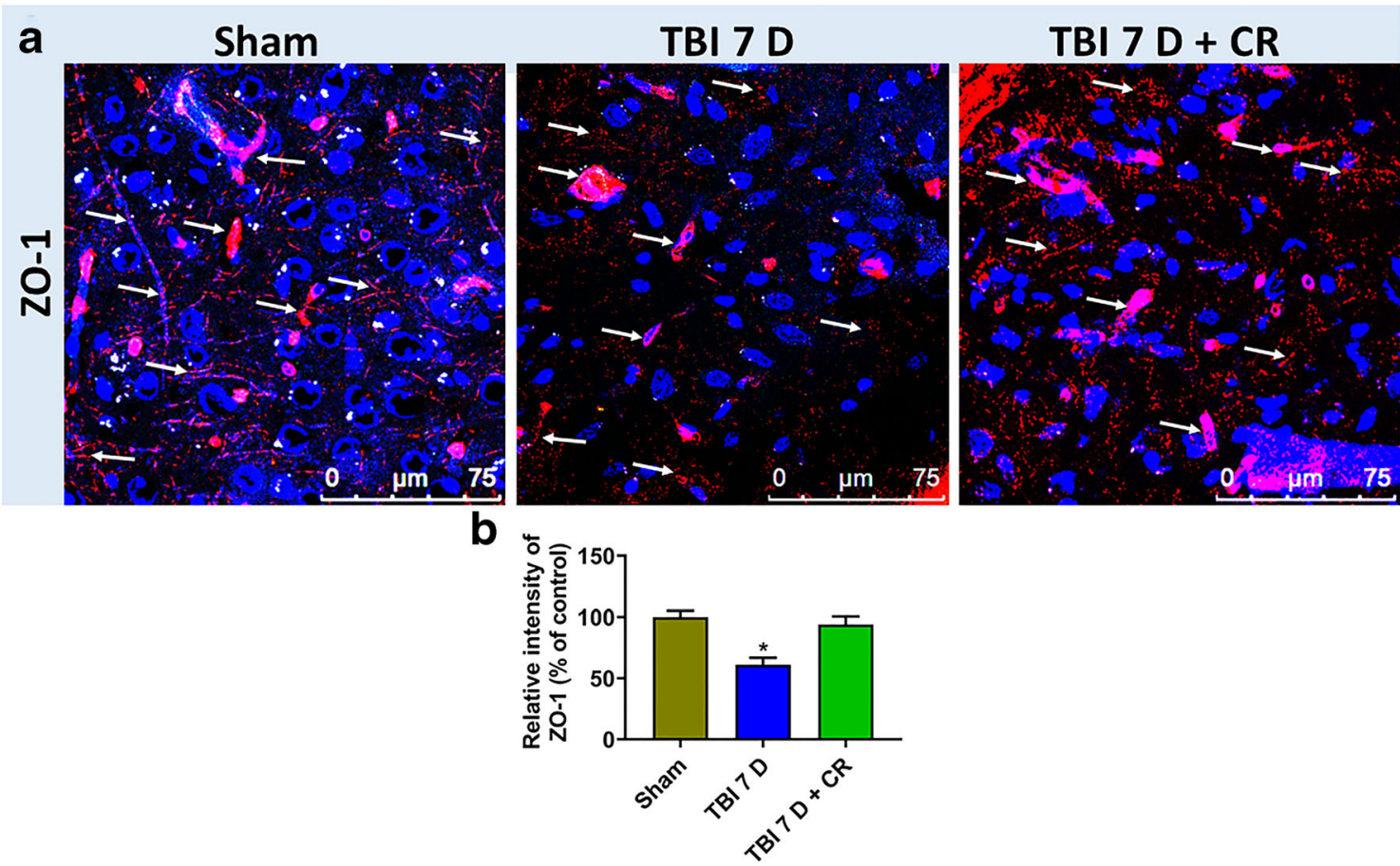

Fig. 3 Closed head weight-drop-induced TBI affects the ZO-1 level in the brain. ZO-1 level was analyzed in the frozen sections $(20 \mu \mathrm{m})$ of brains after 7 days of weight-drop-induced TBI, cromolyn treated mice, and sham control mice brains ( $n=3$ mice/group) by immunofluorescence staining. Cromolyn was administered $1 \mathrm{~h}$ before TBI and 1 day after TBI. Images were captured using a confocal microscope. Representative images and immunoreactivity intensity (a) and bar graph (b) shows the

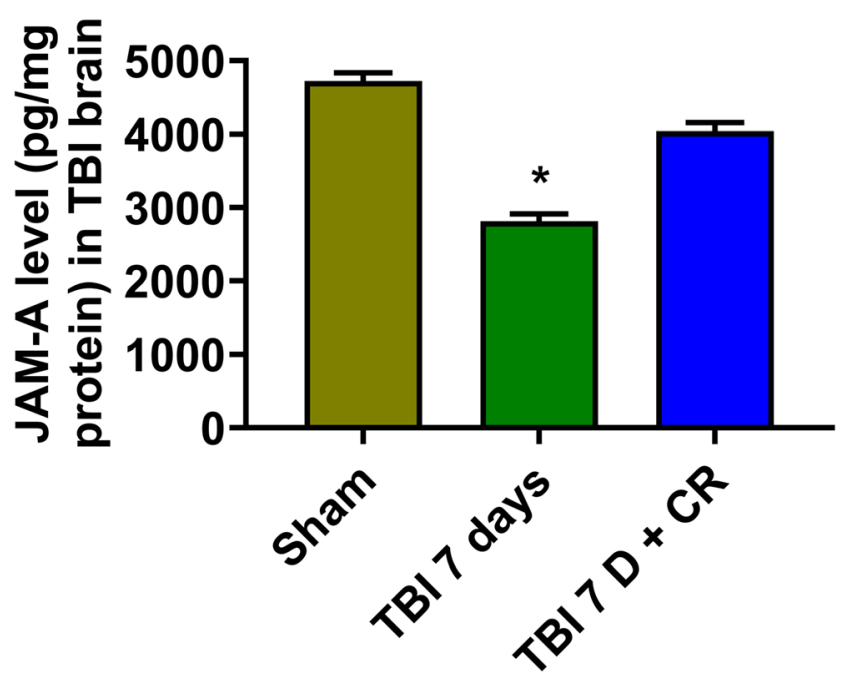

Fig. 4 Cromolyn drug administration inhibits acute TBI-induced decrease of JAM-A level in mice brain. JAM-A level was quantified by ELISA in the brain tissue lysates of sham control mice, 7 days after TBI mice, and 7 days TBI mice administered with cromolyn $(n=3$ mice/group). Results show a significantly decreased level of JAM-A as compared with sham control mice brain $\left({ }^{*} p<0.05\right.$, sham control vs. TBI). Further, the administration of mast cell inhibitor drug cromolyn inhibited TBI-induced decrease of JAM-A as compared with nontreated TBI mice brains indicating its neuroprotective effect in these mice. 7 D, 7 days; CR, cromolyn derangement/decreased level of ZO-1 (arrows) in 7 days of acute TBI brains as compared with sham control brains $(* p<0.05$, sham control vs. TBI). Cromolyn administration in TBI mice prevented TBI-associated decrease of the ZO-1 level. The cellular nuclei were stained with DAPI. Photomicrographs original magnifications $=\times 630.7 \mathrm{D}, 7$ days; $\mathrm{CR}$, cromolyn

brains as compared with sham control mice brains. Further, the administration of mast cell stabilizer (inhibitor) drug cromolyn significantly suppressed the decrease of PDGFR- $\beta$, JAM-A, and ZO-1 levels in TBI mice, indicating the beneficial effects of cromolyn drug in reducing TBIassociated neuroinflammatory response and brain damage. In this preliminary study, we first confirmed TBI-induced activation of astrocytes, indicating astrogliosis and reactive astrocytes, and decreased neurons in the contusion/impact cortical area in the brain. Additionally, mice subjected to acute TBI did not fully recognize the novel object indicating the spatial memory performance is impaired as determined by the NOR test. The brain swelling/edema due to TBI could contribute to these behavioral and memory abnormalities. In the present study, we have selected closed head weight-dropinduced TBI since this induces mild TBI that simulate road accidents, falls, concussive head trauma, and domestic violence activities. Histopathological alterations, immune and inflammatory responses, and behavioral disorders vary in animals, as there are differences in the TBI-induction procedures and the type of animals used (Ma et al. 2019; Zvejniece et al. 2019). Thus, an in-depth understanding of the molecular and ultrastructural mechanism of TBI pathogenesis, especially microvascular and tight junction protein alterations, is essential to develop an effective precision-targeted therapeutic agent 
for acute TBI that could prevent long-term consequences of TBI.

$\mathrm{BBB}$ is considered a therapeutic target for TBI (Abrahamson and Ikonomovic 2020; Jing et al. 2020; Kempuraj et al. 2017; Shen et al. 2019; Thal and Neuhaus 2014). Endothelial cells are connected by many tight junction proteins that form $\mathrm{BBB}$ and regulate the rate of microvascular permeability in the brain. JAM-A and ZO-1 form a tight junction and prevent/regulate BBB permeability in the brain. TBI can cause pericyte damage and loss (Bhowmick et al. 2019). Derangements or down-regulation of tight junction proteins and pericytes can increase $\mathrm{BBB}$ permeability and can cause the formation of edema in the affected region of the brain. A previous study has shown that neurotrauma, including fluid percussion injury (FPI), can decrease tight junction proteins such as JAM-A, ZO-1, occludin, and claudin-5, as well as pericyte markers PDGFR- $\beta$, NG2, and CD13 after $24 \mathrm{~h}$ of brain injury in mice (Bhowmick et al. 2019). Another previous study showed decreased or derangement of tight junction proteins such as JAM-A causes BBB breach and edema $12 \mathrm{~h}$ after brain injury in rats (Yeung et al. 2008). Controlled blunt head trauma in rats and controlled cortical impact (CCI) TBI in mice show decreased ZO-1 level and induced BBB breach (Blixt et al. 2015; Liu et al. 2017). These findings in neurotrauma corroborate our present observation of the derangement or down-regulation of JAM-A and ZO-1 in 7 days after TBI in mice brains. In the present study, in addition to immunofluorescence staining, we have also quantified the JAM-A level by ELISA and show decreased level in the acute TBI brains. All these findings indicate that JAM-A and ZO-1 are necessary for the integrity of tight junction in the brain. A study has shown irregular distribution or increased clustered PDGFR- $\beta$ in degenerating brain areas after fluid percussioninduced TBI (Kyyriainen et al. 2017). These PDGFR- $\beta$ positive migrating cells or PDGFR- $\beta$ positive cells could participate in tissue repair process after the brain injury by secreting the chemokine (C-C motif) ligand 2 (CCL2) (Duan et al. 2018; Kyyriainen et al. 2017; Rafalski et al. 2018).

Mast cells produce many anti-inflammatory and proinflammatory cytokines/chemokines and other molecules that regulate innate and acquired immune response (Arac et al. 2019; Elieh Ali Komi et al. 2019; Kempuraj et al. 2019a). The balance and interrelationship between pro and anti-inflammatory molecules play an essential role in this innate and acquired immune regulation, including in the CNS (Forsythe 2019; Franza et al. 2019; Gallenga et al. 2019). Moreover, both pro and anti-inflammatory cytokines and chemokines can act on mast cells. Viral activation of mast cells can release IL-1, IL-6, and IL-33 and exacerbate inflammation and endothelial functions in the lung and central nervous system in Coronavirus disease 2019 (COVID-19) (Kempuraj et al. 2020b; Kritas et al. 2020). We have previously shown that IL-1 can activate mast cells and selective release of IL-6, which is implicated in COVID-19 (Kandere-Grzybowska et al. 2003; Kempuraj et al. 2020b). IL-1 enhances endothelial cell-leukocyte adhesion, causes inflammation, and the formation of microthrombi through thromboxane A1 production in COVID-19 patients (Conti et al. 2020). The severity of TBI is mainly dependent on the extent of edema than the primary injury itself. Neurovascular unit damage can cause cognitive impairment in neuroinflammatory disorders, including TBI, as observed in the present study. We and others have reported that brain mast cell, including meningeal mast cell activation mediated inflammatory mediators, are implicated in neuroinflammatory response and $\mathrm{BBB} /$ tight junction protein disruption in acute neurotrauma/TBI/stroke in mice (Arac et al. 2019; Kempuraj et al. 2020; McKittrick et al. 2015; Nasr et al. 2019; Ribatti 2015; Rodrigues and Granger 2015; Skaper et al. 2014).

Thus mast cells could be a therapeutic target for neuroprotective strategy (Kempuraj et al. 2019a; Li et al. 2017; Ocak et al. 2018). Previous studies have shown that administration of mast cell inhibitor drug cromolyn suppresses neuroinflammation, brain damage, BBB stability, and improves cognitive disorder after neurotrauma in animals (Jin et al. 2007; Mattila et al. 2011; McKittrick et al. 2015; Valle-Dorado et al. 2015; Zhang et al. 2016). Previous studies suggested that cromolyn treatment provided neuroprotective effects by promoting microglial phagocytosis, reducing proinflammatory microglial state, and increasing the anti-inflammatory microglial state, and inhibiting the severity of disease (Granucci et al. 2019; Zhang et al. 2018). One recent study indicates that cromolyn decreases sensorimotor dysfunction and hippocampal damage in severe TBI in rats (SegoviaOropeza et al. 2020). Therefore, in this short study, we have evaluated cromolyn drug in the present study and demonstrate its beneficial protective effects after acute TBI in mice. In this current study, we administered cromolyn pre (prophylactic) and post (therapeutic) TBI, and the observed effects could be due to both these combined effects. Our future studies will focus on the blood vessel, microglial phenotypes/ Iba1, endothelial cell markers, and the therapeutic effects of cromolyn in TBI mice. TBI directly cause primary damage to the neurovascular unit in the brain. The primary brain damage could lead to immediate and or late neuroimmune and neuroinflammatory responses associated with activation of microglia, astrocytes, and mast cells in the meninges and in the brain parenchyma. The activated glial cells and neurons secrete neuroinflammatory mediators that can further act on glial cells, neurons, endothelial cells, and pericytes in a vicious cycle. This vicious reaction causes increased $\mathrm{BBB}$ permeability, reduced tight junction proteins, and its derangements and edema formation in the brain. This vicious cycle upregulates neuroinflammatory response, neuronal death, and secondary brain damage after TBI. 


\section{Conclusion}

Weight-drop-induced acute TBI causes neuroinflammatory response and $\mathrm{BBB} /$ tight junction proteins disruption in the brain. Cromolyn administration could protect acute TBIinduced brain damage and its long-time consequences of secondary brain damage. Therefore, further in-depth quantitative and neuroimmunological studies are needed to better understand the precise pathways and cells involved in the neuroprotective, neurodegenerative, and neurotoxic mechanisms in closed head acute TBI. Our future studies will focus on the quantification of tight junction proteins and novel therapeutic options for TBI.

Funding Research was sponsored by the Leonard Wood Institute in cooperation with the U.S. Army Research Laboratory and was accomplished under Cooperative Agreement Number W911NF-14-2-0034. The views and conclusions contained in this document are those of the authors and should not be interpreted as representing the official policies, either expressed or implied, of the Leonard Wood Institute, the Army Research Laboratory or the U.S. Government. The U.S. Government is authorized to reproduce and distribute reprints for Government purposes, notwithstanding any copyright notation hereon. The authors express their gratitude for the Acute Effects of Neurotrauma Consortium in assisting and coordinating the conduct of this project at Fort Leonard Wood. This research was also supported by NIH grant AG048205 and the VA Research Career Scientist Award to Asgar Zaheer.

Data Availability Data are available from the corresponding authors.

\section{Compliance with Ethical Standards}

Conflict of Interest The authors have no conflict of interest to report.

Ethical Approval This study was conducted "according to the recommendations in the guide for the care and use of laboratory animals of the National Institutes of Health (NIH) and the approval of the committee on the Ethics of Animal Experiments of the University of Missouri (Columbia, MO).'”

\section{References}

Abrahamson EE, Ikonomovic MD (2020) Brain injury-induced dysfunction of the blood brain barrier as a risk for dementia. Exp Neurol 328:113257

Ahmed ME, Selvakumar GP, Kempuraj D, Thangavel R, Mentor S, Dubova I, Raikwar SP, Zaheer S, Iyer S, Zaheer A (2019) Synergy in disruption of mitochondrial dynamics by Abeta (1-42) and glia maturation factor (GMF) in SH-SY5Y cells is mediated through alterations in fission and fusion proteins. Mol Neurobiol 56:6964-6975

Ahmed ME, Selvakumar GP, Kempuraj D, Raikwar SP, Thangavel R, Bazley K, Wu K, Khan O, Khan A, Zaheer S, Iyer S, Burton C, James D, Zaheer A (2020a) Glia maturation factor (GMF) regulates microglial expression phenotypes and the associated neurological deficits in a mouse model of traumatic brain injury. Mol Neurobiol. https://doi.org/10.1007/s12035-020-02040-y

Ahmed ME, Selvakumar GP, Kempuraj D, Raikwar SP, Thangavel R, Bazley K, Wu K, Khan O, Kukulka K, Bussinger B, Dubova I,
Zaheer SA, Govindarajan R, Iyer SS, Burton C, James D, Zaheer A (2020b) Neuroinflammation mediated by GMF exacerbates neuronal injury in an in vitro model of traumatic brain injury. $\mathrm{J}$ Neurotrauma 37(14):1645-1655

Akamatsu Y, Hanafy KA (2020) Cell death and recovery in traumatic brain injury. Neurotherapeutics 17(2):446-456

Arac A, Grimbaldeston MA, Galli SJ, Bliss TM, Steinberg GK (2019) Meningeal mast cells as key effectors of stroke pathology. Front Cell Neurosci 13:126

Bader M, Li Y, Tweedie D, Shlobin NA, Bernstein A, Rubovitch V, Tovar YRLB, DiMarchi RD, Hoffer BJ, Greig NH, Pick CG (2019) Neuroprotective effects and treatment potential of incretin mimetics in a murine model of mild traumatic brain injury. Front Cell Dev Biol 7:356

Bhowmick S, D’Mello V, Caruso D, Wallerstein A, Abdul-Muneer PM (2019) Impairment of pericyte-endothelium crosstalk leads to bloodbrain barrier dysfunction following traumatic brain injury. Exp Neurol 317:260-270

Blixt J, Svensson M, Gunnarson E, Wanecek M (2015) Aquaporins and blood-brain barrier permeability in early edema development after traumatic brain injury. Brain Res 1611:18-28

Charkviani M, Muradashvili N, Lominadze D (2019) Vascular and nonvascular contributors to memory reduction during traumatic brain injury. Eur J Neurosci 50:2860-2876

Choi YK, Maki T, Mandeville ET, Koh SH, Hayakawa K, Arai K, Kim YM, Whalen MJ, Xing C, Wang X, Kim KW, Lo EH (2016) Dual effects of carbon monoxide on pericytes and neurogenesis in traumatic brain injury. Nat Med 22:1335-1341

Conti P, Caraffa A, Gallenga CE, Ross R, Kritas SK, Frydas I, Younes A, Di Emidio P, Ronconi G, Toniato E (2020) IL-1 induces throboxane-A2 (TxA2) in COVID-19 causing inflammation and micro-thrombi: inhibitory effect of the IL-1 receptor antagonist (IL-1Ra). J Biol Regul Homeost Agents 34(5). https://doi.org/10. 23812/20-34-4EDIT-65

Crupi R, Cordaro M, Cuzzocrea S, Impellizzeri D (2020) Management of traumatic brain injury: from present to future. Antioxidants (Basel) 9(4):297

Dinet V, Petry KG, Badaut J (2019) Brain-immune interactions and neuroinflammation after traumatic brain injury. Front Neurosci 13:1178

Duan L, Zhang XD, Miao WY, Sun YJ, Xiong G, Wu Q, Li G, Yang P, Yu H, Li H, Wang Y, Zhang M, Hu LY, Tong X, Zhou WH, Yu X (2018) PDGFRbeta cells rapidly relay inflammatory signal from the circulatory system to neurons via chemokine CCL2. Neuron 100(183-200):e188

Elieh Ali Komi D, Wohrl S, Bielory L (2019) Mast cell biology at molecular level: a comprehensive review. Clin Rev Allergy Immunol 58(3):342-365

Forsythe P (2019) Mast cells in neuroimmune interactions. Trends Neurosci 42:43-55

Franza L, Carusi V, Altamura S, Caraffa A, Gallenga CE, Kritas SK, Ronconi G, Conti P, Pandolfi F (2019) Interrelationship between inflammatory cytokines (IL-1, IL-6, IL-33, IL-37) and acquired immunity. J Biol Regul Homeost Agents 33:1321-1326

Gallenga CE, Pandolfi F, Caraffa A, Kritas SK, Ronconi G, Toniato E, Martinotti S, Conti P (2019) Interleukin-1 family cytokines and mast cells: activation and inhibition. J Biol Regul Homeost Agents 33:16

Granucci EJ, Griciuc A, Mueller KA, Mills AN, Le H, Dios AM, McGinty D, Pereira J, Elmaleh D, Berry JD, Paganoni S, Cudkowicz ME, Tanzi RE, Sadri-Vakili G (2019) Cromolyn sodium delays disease onset and is neuroprotective in the SOD1(G93A) mouse model of amyotrophic lateral sclerosis. Sci Rep 9:17728

He JT, Zhao X, Xu L, Mao CY (2020) Vascular risk factors and Alzheimer's disease: blood-brain barrier disruption, metabolic syndromes, and molecular links. J Alzheimers Dis 73:39-58 
Jin Y, Silverman AJ, Vannucci SJ (2007) Mast cell stabilization limits hypoxic-ischemic brain damage in the immature rat. Dev Neurosci 29:373-384

Jin Y, Silverman AJ, Vannucci SJ (2009) Mast cells are early responders after hypoxia-ischemia in immature rat brain. Stroke 40:3107-3112

Jing Y, Yang DX, Wang W, Yuan F, Chen H, Ding J, Geng Z, Tian HL (2020) Aloin protects against blood-brain barrier damage after traumatic brain injury in mice. Neurosci Bull 36(6):625-638

Jullienne A, Obenaus A, Ichkova A, Savona-Baron C, Pearce WJ, Badaut J (2016) Chronic cerebrovascular dysfunction after traumatic brain injury. J Neurosci Res 94:609-622

Kandere-Grzybowska K, Letourneau R, Kempuraj D, Donelan J, Poplawski S, Boucher W, Athanassiou A, Theoharides TC (2003) IL-1 induces vesicular secretion of IL-6 without degranulation from human mast cells. J Immunol 171:4830-4836

Kempuraj D, Selvakumar GP, Thangavel R, Ahmed ME, Zaheer S, Raikwar SP, Iyer SS, Bhagavan SM, Beladakere-Ramaswamy S, Zaheer A (2017) Mast cell activation in brain injury, stress, and post-traumatic stress disorder and Alzheimer's disease pathogenesis. Front Neurosci 11:703

Kempuraj D, Selvakumar GP, Thangavel R, Ahmed ME, Zaheer S, Kumar KK, Yelam A, Kaur H, Dubova I, Raikwar SP, Iyer SS, Zaheer A (2018a) Glia maturation factor and mast cell-dependent expression of inflammatory mediators and proteinase activated Receptor-2 in neuroinflammation. J Alzheimers Dis 66(3):11171129

Kempuraj D, Thangavel R, Selvakumar GP, Ahmed ME, Zaheer S, Raikwar SP, Zahoor H, Saeed D, Dubova I, Giler G, Herr S, Iyer SS, Zaheer A (2018b) Mast cell proteases activate astrocytes and glia-neurons and release interleukin-33 by activating p38 and ERK1/2 MAPKs and NF-kappaB. Mol Neurobiol 56(3):1681-1693

Kempuraj D, Ahmed ME, Selvakumar GP, Thangavel R, Dhaliwal AS, Dubova I, Mentor S, Premkumar K, Saeed D, Zahoor H, Raikwar SP, Zaheer S, Iyer SS, Zaheer A (2019a) Brain injury-mediated neuroinflammatory response and Alzheimer's disease. Neuroscientist 26:134-155

Kempuraj D, Mentor S, Thangavel R, Ahmed ME, Selvakumar GP, Raikwar SP, Dubova I, Zaheer S, Iyer SS, Zaheer A (2019b) Mast cells in stress, pain, blood-brain barrier, neuroinflammation and Alzheimer's disease. Front Cell Neurosci 13:54

Kempuraj D, Ahmed ME, Selvakumar GP, Thangavel R, Raikwar SP, Zaheer SA, Iyer SS, Burton C, James D, Zaheer A (2020) Mast cell activation, neuroinflammation, and tight junction protein derangement in acute traumatic brain injury. Mediat Inflamm 2020: 4243953-4243912. https://doi.org/10.1155/2020/4243953

Kempuraj D, Ahmed ME, Selvakumar GP, Thangavel R, Raikwar SP, Zaheer SA, Iyer SS, Burton C, James D, Zaheer A (2020a) Psychological stress-induced immune response and risk of Alzheimer's disease in veterans from operation enduring freedom and operation Iraqi freedom. Clin Ther 42:974-982

Kempuraj D, Selvakumar GP, Ahmed ME, Raikwar SP, Thangavel R, Khan A, Zaheer SA, Iyer SS, Burton C, James D, Zaheer A (2020b) COVID-19, mast cells, cytokine storm, psychological stress, and neuroinflammation. Neuroscientist. https://doi.org/10.1177/ 1073858420941476

Khellaf A, Khan DZ, Helmy A (2019) Recent advances in traumatic brain injury. J Neurol 266(11):2878-2889

Kritas SK, Ronconi G, Caraffa A, Gallenga CE, Ross R, Conti P (2020) Mast cells contribute to coronavirus-induced inflammation: new anti-inflammatory strategy. J Biol Regul Homeost Agents 34(1):914

Kyyriainen J, Ekolle Ndode-Ekane X, Pitkanen A (2017) Dynamics of PDGFRbeta expression in different cell types after brain injury. Glia 65:322-341
Ladak AA, Enam SA, Ibrahim MT (2019) A review of the molecular mechanisms of traumatic brain injury. World Neurosurg 131:126132

Li N, Zhang X, Dong H, Hu Y, Qian Y (2017) Bidirectional relationship of mast cells-neurovascular unit communication in neuroinflammation and its involvement in POCD. Behav Brain Res 322:60-69

Liu YL, Xu ZM, Yang GY, Yang DX, Ding J, Chen H, Yuan F, Tian HL (2017) Sesamin alleviates blood-brain barrier disruption in mice with experimental traumatic brain injury. Acta Pharmacol Sin 38: $1445-1455$

Ma X, Aravind A, Pfister BJ, Chandra N, Haorah J (2019) Animal models of traumatic brain injury and assessment of injury severity. Mol Neurobiol 56:5332-5345

Mattila OS, Strbian D, Saksi J, Pikkarainen TO, Rantanen V, Tatlisumak T, Lindsberg PJ (2011) Cerebral mast cells mediate blood-brain barrier disruption in acute experimental ischemic stroke through perivascular gelatinase activation. Stroke 42:3600-3605

McKee AC, Robinson ME (2014) Military-related traumatic brain injury and neurodegeneration. Alzheimers Dement 10:S242-S253

McKittrick CM, Lawrence CE, Carswell HV (2015) Mast cells promote blood brain barrier breakdown and neutrophil infiltration in a mouse model of focal cerebral ischemia. J Cereb Blood Flow Metab 35: 638-647

Milman A, Rosenberg A, Weizman R, Pick CG (2005) Mild traumatic brain injury induces persistent cognitive deficits and behavioral disturbances in mice. J Neurotrauma 22:1003-1010

Moretti R, Chhor V, Bettati D, Banino E, De Lucia S, Le Charpentier T, Lebon S, Schwendimann L, Pansiot J, Rasika S, Degos V, Titomanlio L, Gressens P, Fleiss B (2016) Contribution of mast cells to injury mechanisms in a mouse model of pediatric traumatic brain injury. J Neurosci Res 94:1546-1560

Nasr IW, Chun Y, Kannan S (2019) Neuroimmune responses in the developing brain following traumatic brain injury. Exp Neurol 320:112957

Nation DA, Sweeney MD, Montagne A, Sagare AP, D’Orazio LM, Pachicano M, Sepehrband F, Nelson AR, Buennagel DP, Harrington MG, Benzinger TLS, Fagan AM, Ringman JM, Schneider LS, Morris JC, Chui HC, Law M, Toga AW, Zlokovic BV (2019) Blood-brain barrier breakdown is an early biomarker of human cognitive dysfunction. Nat Med 25:270-276

Ocak U, Ocak PE, Wang A, Zhang JH, Boling W, Wu P, Mo J, Zhang T, Huang L (2018) Targeting mast cell as a neuroprotective strategy. Brain Inj:1-11

Rafalski VA, Merlini M, Akassoglou K (2018) Pericytes: the brain's very first responders? Neuron 100:11-13

Raikwar SP, Thangavel R, Dubova I, Selvakumar GP, Ahmed ME, Kempuraj D, Zaheer SA, Iyer SS, Zaheer A (2018) Targeted gene editing of glia maturation factor in microglia: a novel Alzheimer's disease therapeutic target. Mol Neurobiol 56(1):378-393

Raikwar SP, Thangavel R, Ahmed ME, Selvakumar GP, Kempuraj D, Wu K, Khan O, Bazley K, Bussinger B, Kukulka K, Zaheer S, Iyer SS, Govindarajan R, Burton C, James D, Zaheer A (2020) Real-time noninvasive bioluminescence, ultrasound and photoacoustic imaging in NFkappaB-RE-Luc transgenic mice reveal glia maturation factor-mediated immediate and sustained spatio-temporal activation of NFkappaB signaling post-traumatic brain injury in a genderspecific manner. Cell Mol Neurobiol. https://doi.org/10.1007/ s10571-020-00937-9

Ribatti D (2015) The crucial role of mast cells in blood-brain barrier alterations. Exp Cell Res 338:119-125

Rodrigues SF, Granger DN (2015) Blood cells and endothelial barrier function. Tissue Barriers 3:e978720

Segovia-Oropeza M, Santiago-Castaneda C, Orozco-Suarez SA, Concha L, Rocha L (2020) Sodium cromoglycate decreases sensorimotor impairment and hippocampal alterations induced by severe 
traumatic brain injury in rats. J Neurotrauma. https://doi.org/10. 1089/neu.2019.6975

Selvakumar GP, Iyer SS, Kempuraj D, Ahmed ME, Thangavel R, Dubova I, Raikwar SP, Zaheer S, Zaheer A (2018) Molecular association of glia maturation factor with the autophagic machinery in rat dopaminergic neurons: a role for endoplasmic reticulum stress and MAPK activation. Mol Neurobiol 56(6):3865-3881

Selvakumar GP, Ahmed ME, Iyer SS, Thangavel R, Kempuraj D, Raikwar SP, Bazley K, Wu K, Khan A, Kukulka K, Bussinger B, Zaheer S, Burton C, James D, Zaheer A (2020) Absence of glia maturation factor protects from axonal injury and motor behavioral impairments after traumatic brain injury. Exp Neurobiol 29:230 248

Sengillo JD, Winkler EA, Walker CT, Sullivan JS, Johnson M, Zlokovic BV (2013) Deficiency in mural vascular cells coincides with bloodbrain barrier disruption in Alzheimer's disease. Brain Pathol 23: 303-310

Shen J, Xin W, Li Q, Gao Y, Yuan L, Zhang J (2019) Methylene blue reduces neuronal apoptosis and improves blood-brain barrier integrity after traumatic brain injury. Front Neurol 10:1133

Shishido H, Ueno M, Sato K, Matsumura M, Toyota Y, Kirino Y, Tamiya T, Kawai N, Kishimoto Y (2019) Traumatic brain injury by weight-drop method causes transient amyloid-beta deposition and acute cognitive deficits in mice. Behav Neurol 2019:3248519

Sivandzade F, Alqahtani F, Cucullo L (2020) Traumatic brain injury and blood-brain barrier (BBB): underlying pathophysiological mechanisms and the influence of cigarette smoking as a premorbid condition. Int J Mol Sci 21(8):2721

Skaper SD, Facci L, Giusti P (2014) Neuroinflammation, microglia and mast cells in the pathophysiology of neurocognitive disorders: a review. CNS Neurol Disord Drug Targets 13:1654-1666

Tadepalli SA, Bali ZK, Bruszt N, Nagy LV, Amrein K, Fazekas B, Buki A, Czeiter E, Hernadi I (2020) Long-term cognitive impairment without diffuse axonal injury following repetitive mild traumatic brain injury in rats. Behav Brain Res 378:112268
Thal SC, Neuhaus W (2014) The blood-brain barrier as a target in traumatic brain injury treatment. Arch Med Res 45:698-710

Thangavel R, Bhagavan SM, Ramaswamy SB, Surpur S, Govindarajan R, Kempuraj D, Zaheer S, Raikwar S, Ahmed ME, Selvakumar GP, Iyer SS, Zaheer A (2018) Co-expression of glia maturation factor and apolipoprotein E4 in Alzheimer's disease brain. J Alzheimers Dis 61:553-560

Valle-Dorado MG, Santana-Gomez CE, Orozco-Suarez SA, Rocha L (2015) The mast cell stabilizer sodium cromoglycate reduces histamine release and status epilepticus-induced neuronal damage in the rat hippocampus. Neuropharmacology 92:49-55

Yeung D, Manias JL, Stewart DJ, Nag S (2008) Decreased junctional adhesion molecule-A expression during blood-brain barrier breakdown. Acta Neuropathol 115:635-642

Zhang S, Dong H, Zhang X, Li N, Sun J, Qian Y (2016) Cerebral mast cells contribute to postoperative cognitive dysfunction by promoting blood brain barrier disruption. Behav Brain Res 298:158-166

Zhang C, Griciuc A, Hudry E, Wan Y, Quinti L, Ward J, Forte AM, Shen X, Ran C, Elmaleh DR, Tanzi RE (2018) Cromolyn reduces levels of the Alzheimer's disease-associated amyloid beta-protein by promoting microglial phagocytosis. Sci Rep 8:1144

Zohar O, Schreiber S, Getslev V, Schwartz JP, Mullins PG, Pick CG (2003) Closed-head minimal traumatic brain injury produces longterm cognitive deficits in mice. Neuroscience 118:949-955

Zvejniece L, Stelfa G, Vavers E, Kupats E, Kuka J, Svalbe B, Zvejniece B, Albert-Weissenberger C, Siren AL, Plesnila N, Dambrova M (2019) Skull fractures induce neuroinflammation and worsen outcomes after closed head injury in mice. J Neurotrauma 37(2):295304

Zysk M, Clausen F, Aguilar X, Sehlin D, Syvanen S, Erlandsson A (2019) Long-term effects of traumatic brain injury in a mouse model of Alzheimer's disease. J Alzheimers Dis 72(1):161-180

Publisher's Note Springer Nature remains neutral with regard to jurisdictional claims in published maps and institutional affiliations. 